\title{
Erratum to: The electronic structure and intervalley coupling of artificial and genuine graphene superlattices
}

\author{
Pilkwang Kim and Cheol-Hwan Park $(\varangle)$ \\ Department of Physics and Astronomy, Seoul National University, Seoul 08826, Republic of Korea \\ (C) Tsinghua University Press and Springer-Verlag Berlin Heidelberg 2016
}

\section{Erratum to}

Nano Research 2016, 9(4): 1101-1115

DOI 10.1007/s12274-016-1004-2

The original version of this article unfortunately failed to cite four relevant papers (Refs. [53-56]). References $[53,54]$ report on a universal tight binding Hamiltonian of graphene. With an adjustable hopping parameter, the Hamiltonian successfully describes the merging of Dirac points and opening up of a band gap. References $[55,56]$ report that the energy spectrum of a twisted bilayer graphene exhibits merging and splitting of the Dirac points.

The first sentence in the third paragraph of Section 3.2 in page 1109 , instead of "... adopting single-valley approximation $[45,46,50]$ ", should read "...adopting single-valley approximation [45, 46, 50], although there have been studies on universal tight-binding Hamiltonians on graphene and on twisted bilayer graphene predicting similar features [53-56]."

The second last sentence of Section 3.2, instead of "... in the previous studies adopting single-valley approximation [46, 53]:", should read “... in the previous studies adopting single-valley approximation [46, 57]:".

The online version of the original article can be found at http://dx.doi.org/10.1007/s12274-016-1004-2.
The last part of References should be, instead of

[53] Park, C.-H.; Tan, L. Z.; Louie, S. G. Theory of the electronic and transport properties of graphene under a periodic electronic or magnetic field. Phys. E 2011, 43, 651-656.

should read

[53] Montambaux, G.; Piéchon, F.; Fuchs, J.-N.; Goerbig, M. O. Merging of Dirac points in a two-dimensional crystal. Phys. Rev. B 2009, 80, 153412.

[54] Montambaux, G.; Piéchon, F.; Fuchs, J.-N.; Goerbig, M. O. A universal Hamiltonian for motion and merging of Dirac points in a two-dimensional crystal. Eur. Phys. J. B 2009, $72,509$.

[55] de Gail, R.; Fuchs, J.-N.; Goerbig, M. O.; Piéchon, F.; Montambaux, G. Manipulation of Dirac points in graphenelike crystals. Physica B: Physics of Condensed Matter 2012, 407, 1948.

[56] Goerbig, M. O.; Montambaux, G. Dirac fermions in condensed matter and beyond. "Matière de Dirac", Séminaire Poincaré 2014, Vol. XVIII.

[57] Park, C.-H.; Tan, L. Z.; Louie, S. G. Theory of the electronic and transport properties of graphene under a periodic electronic or magnetic field. Phys. E 2011, 43, 651-656.

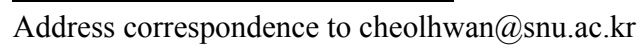

